\title{
Geleitwort von Prof. Dr. Lothar H. Wieler
}

\section{„Public Health kompakt"}

Public Health, verstanden als die öffentliche Sorge um die Gesundheit aller, ist ein sehr dynamisches Gebiet. Allein die Frage, welche Akteure welchen Beitrag zu wessen Gesundheit leisten können oder auch sollen, ist aktuell in Deutschland heiß diskutiert. Das spiegelt sich auch in der Weiterentwicklung dieses Buches von der ersten über die zweite bis hin zur aktuell vorgelegten dritten Ausgabe wider. Nicht nur, dass die große Nachfrage erfreulicherweise eine Neuauflage erforderlich gemacht hat, auch die Rahmenbedingungen für Public Health unterliegen einem stetigen Wandel.

So werden neuere Konzepte von Public Health in dieses Buch aufgenommen und diskutiert. Die zunehmende Globalisierung von Gesundheitsrisiken bedingt, dass auch Public Health global gedacht und zu „Global Health“ wird. Der zusätzliche, neue Aspekt, dass mit den großen Zuwanderungsströmen nach Europa in den letzten Jahren diese Globalisierung sozusagen vor der Haustür stattfindet, findet seinen Niederschlag. Die Themen Migration und Flucht, die Sorge um die Gesundheit Geflüchteter und erforderliche Maßnahmen sind neue Themen dieser Ausgabe. Im Zuge des demographischen Wandels und der Alterung der Gesellschaft gewinnt die Gesundheit bzw. die Gesunderhaltung im Lebensverlauf zentrale Bedeutung. Folgerichtig gibt es neu in dieser Ausgabe das Kapitel „Gesundheit im Verlauf des Lebens - Life Course Approach to Health“.

Das europäische Regionalbüro der WHO definiert 10 zentrale Handlungsfelder von Public Health, die folgende Aspekte umfassen: Die Surveillance von Gesundheit und Lebensqualität in der Bevölkerung, die Befähigung zu raschen Reaktionen bei Gesundheitsgefahren und Notlagen, den Gesundheitsschutz unter Einschluss von Umwelt, Arbeitsleben und Lebensmittelsicherheit, die Gesundheitsförderung unter Berücksichtigung sozialer Determinanten mit dem Ziel der Herstellung gesundheitlicher Chancengleichheit, Krankheitsprävention und -früherkennung, die Wahrnahme von Steuerungsfunktionen im Bereich Gesundheit, die Sicherstellung einer nachhaltigen Organisation und Finanzierung, eine (angewandte) Public-Health-Forschung, eine wissensbasierte Politikberatung und die Ausbildung von Public-Health-Akteuren in ausreichender Zahl und Kompetenz. Insbesondere zu Letzterem leistet dieses Buch einen hervorragenden Beitrag, indem das Basiswissen, sozusagen das Handwerkszeug von Public Health, aktuell vermittelt wird.

Für alle 10 zentralen Handlungsfelder von Public Health trifft dieses Buch Aussagen, gibt Informationen und Anleitungen zum Handeln. Und das umfasst weit mehr als rein medizinische Aspekte, was zur Folge hat, dass auch der Adressatenund Leserkreis dieses Buches immer größer wird. Es sind schon lange nicht mehr nur Mediziner oder Medizinstudenten, denn Public Health ist multi- und interdisziplinär und braucht ein Netzwerk ganz vieler Akteure. In diesem Sinne freue ich mich 
über diese aktuelle dritte Auflage des „Public Health kompakt“ und wünsche den Herausgebern, dass recht bald eine weitere, vierte folgen muss.

Prof. Dr. Lothar H. Wieler

Präsident

Robert Koch-Institut, Berlin 